\title{
Angular induction is modulated by the orientation of the test segment but not its length
}

\author{
ERNEST GREENE \\ University of Southern California, Los Angeles, California \\ and Neuropsychology Foundation, Los Angeles, California
}

\begin{abstract}
Angular induction is the basic process by which the orientation of line segments can affect the perceived orientation of other segments as well as their alignment. In this laboratory, we have found that the effect on alignment follows approximately linear rules, with the inducing segment having its greatest influence when its orientation is near to that of the segment being judged. Other laboratories, however, have reported peak effects when the relative angle between the two is at $45^{\circ}$, and with the inducing segment being aligned with one of the cardinal axes of the observer. It has been said that the length of the test segment being judged is a critical factor, but the first experiment of the present study shows a similar linear decline of induction strength irrespective of test segment length. The second experiment indicates that the orientation of the test segment relative to the observer modulates the induction to determine the location of peak effects. A two-factor linear model predicts the observed pattern of results.
\end{abstract}

The angular induction that is produced by one line segment can influence the perceived orientation of another segment and can affect the perceived alignment among segments and points. Such induction likely provides the substrate for geometric illusions of angle and directionmost notably, the Poggendorff illusion. To provide adequate experimental control over the variables, the present work was performed with a relatively restricted set of line elements-specifically, a test segment whose collinear relationship was to be judged, and a segment, described as the induction segment, whose orientation and proximity to the test segment served to bias these judgments.

The major issue to be resolved was why some laboratories (see, e.g., Bouma \& Andriessen, 1970; Weintraub \& Krantz, 1971) have reported that the strength of angular induction is greatest where the inducing segment is aligned with the horizontal or vertical axis, whereas results from this laboratory have shown that the effects peak at a close angle irrespective of cardinal axis alignment. Most investigators have used complex stimuli (such as the Poggendorff illusion) that have contained at least two inducing lines, but Greene and Pavlov (1989) found that the same principles applied whether the configuration contained one induction line or two. Further, Bouma and Andriessen (1970) found very clear evidence of a cardinal axis peak in judging the collinearity of a test segment when there was only one induction line, thus obviating the argument that the location of the peak depended on multiple induction stimulus configurations. The fact that we

This research was supported in part by the Neuropsychology Foundation. Correspondence should be addressed to E. Greene, Department of Psychology, University of Southern California, Los Angeles, CA 90089-1061. have gotten results different from Bouma and Andriessen's (1970) is especially puzzling, since the test conditions and the perceptual judgments that they required seem comparable to our own.

One potential explanation for the discrepancy is related to the length of the test segment. We have reported on induction effects with test segments that ranged in length from 6 to $8 \mathrm{~cm}\left(448^{\prime}\right.$ to $596^{\prime}$ of visual angle), and have done pilot work in which the test segment was only $1 \mathrm{~cm}$ $\left(74^{\prime}\right)$. With these stimuli, we have found peak induction effects when the relative angle between the test segment and the induction segment was at $15^{\circ}$ or closer (Greene \& Pavlov, 1989; Greene, in press). Bouma and Andriessen (1970) used 14' and 28' test segments, and they found the strongest effects near $45^{\circ}$, where the induction segment was aligned with or in proximity to a cardinal axis. Wenderoth, Parkinson, and White (1979) reported that the strength of induction, as measured by alignment of the test segment with a dot, decreased as a function of angle for long test segments, but peaked at $45^{\circ}$ when the test segment was short.

To explain peak induction as a function of test segment length, one could formulate a neurobiological model of receptive-field interaction. For example, one might adopt a model which hypothesized that neuron receptive fields were designed to detect very short segments, with the analysis of long segments requiring the combined activity from an entire array of collinear receptive fields (see Andrews, 1967a, 1967b; Bacon \& King-Smith, 1977; Tyler \& Nakayama, 1984; Vassilev \& Penchev, 1976). Each individual field might well have a peak sensitivity to induction at $45^{\circ}$, but the array-acting as a unit-would respond to an induction segment as a linear function of angle, reaching a peak at a closer angle where many or most of the receptive fields in the array were being affected. 
In Experiment 1, we evaluated whether the length of the test segment would determine the strength of induction or the location of peak effects. The results indicated a linear change in strength of effect with an increase in the relative angle between the test and induction segments. Close angles produced the strongest bias, irrespective of the length of the segment being judged. This appears to eliminate test segment length as the basis for Bouma and Andriessen's (1970) finding that induction was strongest when the angle was at $45^{\circ}$.

In a second experiment, we examined whether procedural differences might account for the discrepancy. Specifically, in this laboratory, we define the basic induction effect in terms of the relative angle between the test segment and the induction segment. To this effect, one must add a second factor-namely, the orientation of the test segment relative to the observer (as specified in the section on modeling below). Although each of the two factors appears to be linear within certain ranges, one can combine them to predict biphasic effects with a peak at $45^{\circ}$. The basic method used by Bouma and Andriessen (1970) was to hold the induction line at a constant position and vary the orientation of the test segment, which confounded the effects by changing the relative angle between the two segments and the orientation of the test segment to the observer simultaneously.

In the second experiment, we used Bouma and Andriessen's (1970) procedure for manipulating angle, and found that the strength of induction peaked near $45^{\circ}$ even though the test segments were long. Additionally, the strength and pattern of the induction effects were measured where the test segment was kept at a fixed position and the induction segment was varied, as is our normal method for measuring the process. Values derived from these observations were incorporated into a formal model that was able to predict the effects that had been found with Bouma and Andriessen's (1970) protocols.

\section{EXPERIMENT 1}

In Experiment 1, subjects made collinearity judgments of various lengths of test segments that were placed at $45^{\circ}$ relative to the observer. Most stimulus configurations also included an induction segment, which was placed at a specified angle relative to the test segment in order to bias the collinearity judgment. Figure 1 illustrates the position of the segments and identifies angular relationships.

The goal of Experiment 1 was to determine the strength of bias as a function of the orientation of the induction segment and of the length of the test segment. The task required the subject to place a point across the span of the page at a location that appeared to be "in line" with the test segment. This "free operant" method for judging collinearity has been evaluated by Greene (1993), who has found that the size of the induction bias is a constant angular error irrespective of the distance chosen by the subject.

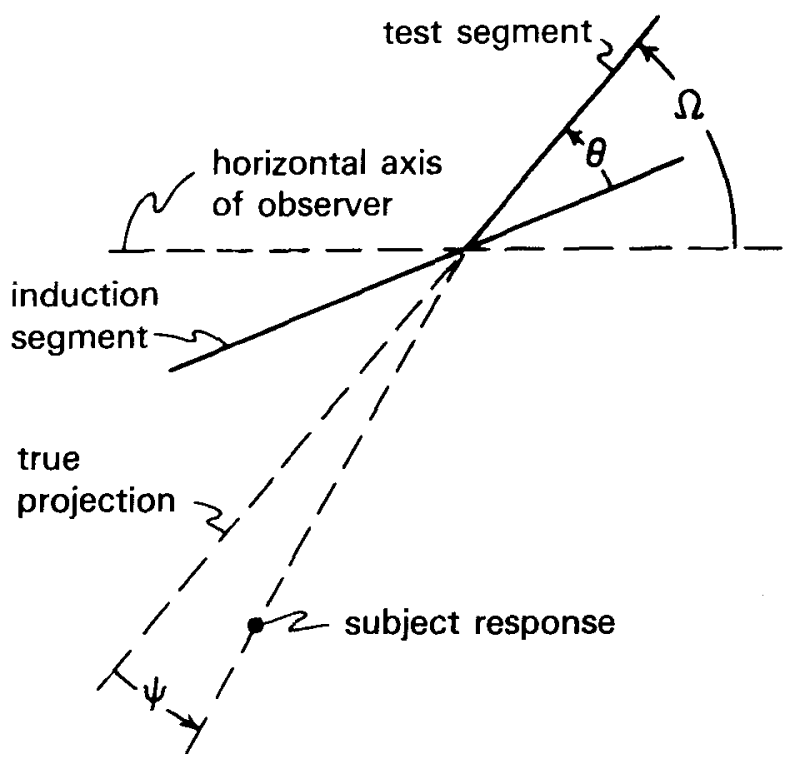

Figure 1. In both experiments, the visual stimulus consisted of two lines - the test and induction segments-which were positioned as illustrated. The orientation of the induction segment $(\theta)$ was defined relative to the test segment, and the orientation of the test segment $(\Omega)$ was defined relative to the horizontal axis of the observerthese $\Omega$ effects being thought equivalent for the vertical axis. The amount of collinearity error $(\psi)$ was measured as an angle from the true projection of the test segment. The sign of angles depended on the page quadrant in which the stimulus was displayed, as described in Methods.

\section{Method}

Stimulus materials. The test set containing the line configurations to be judged was printed on an Apple LaserWriter with a nominal resolution of $300 \mathrm{dpi}$. The line segments were checked with a straightedge, and no distortion could be detected. Segment thickness was 2' of visual angle. This and all other segment dimensions reported here were measured with a dissecting microscope containing a calibrated reticule.

The stimulus display presented the test segment in one quadrant, with the long axis of the page being horizontal relative to the observer. The subject made a collinearity judgment by placing a point across the open space of the page into the opposite quadrant at the location that appeared to be aligned with the test segment. For each page in the set, the test segment was placed at an orientation of $45^{\circ}$ relative to the vertical axis of the page and was aligned with the center. The distance between the page-center and the closest tip of the segment was $7.5^{\circ}$ of visual angle. The fact that the test segment was collinear with the center of the page was not known to the subject, and it was not obvious because of the influence of the induction segment that was present on most of the test pages (as is described below). Across the stimulus set, each stimulus configuration was presented in each of the page quadrants. Prior research from this laboratory has not found the differences in response in the four quadrants to be of practical or theoretical significance, and therefore this factor was not retained as a separate treatment condition.

The first experimental treatment varied the length of the test segment. Each stimulus page contained one of four lengths $-21^{\prime}, 63^{\prime}$, $189^{\prime}$, or $567^{\prime}$ of visual angle-with the segment being positioned in one quadrant as described above. In most cases, the page also 
contained an induction segment with a length of $720^{\prime}$, which was positioned at one of six orientations, with its center touching the tip of the test segment closest to the center of the page. These induction angles, designated as theta, were $15^{\circ}, 30^{\circ}, 45^{\circ}, 60^{\circ}, 75^{\circ}$, and $90^{\circ}$. In the upper right and lower left quadrants, theta was measured clockwise to the orientation of the test segment, and for the other two quadrants, it was measured as a counterclockwise angle. This convention preserved vertical symmetry for the observer, in that a $45^{\circ}$ angle presented a horizontal induction segment irrespective of the quadrant in which it occurred.

The absence of an induction segment was designated as the $0^{\circ}$ condition. Therefore, there were four levels of test segment length, and seven levels of induction angle. The complete test set contained each combination of the treatments for each of the quadrants, for a total of $4 \times 7 \times 4=112$ pages in the test package. These were presented in a different random order for each subject.

Subjects and Test protocols. Eight U.S.C. undergraduates with $20 / 20$ vision (allowing correction by the use of contact lenses) served as the subjects for this experiment. They were naive with respect to the induction phenomenon, and when questioned afterward, they gave no indication that any had deduced the nature of its influence. Each was tested with the aid of a viewing stand and a Lone Ranger mask, which was similar to that used by Weintraub and Brown (1986). The mask and stand served to position the page in a plane that was tangent to the viewing axis of the observer, and with the center of the page at $46 \mathrm{~cm}$ from the eyes. The mask discouraged the subject from tilting the head, because this would result in disruption of the binocular image.

The subjects were given general instructions about the collinearity judgments that were required. They were told to place each page on the viewing stand, to align it with the aid of an edge-stop, and to place a point on the page that was "in line" with the test segment. The general location for placement of the point was described verbally as being "on the other side of the page," and by gesture, a distance of roughly $15^{\circ}-20^{\circ}$ of visual angle from the tip of the test segment was indicated. They were asked to avoid stroking across the page as though a line were being drawn, and thereafter they were allowed to begin and to proceed at their own paces until they had judged each page of the set.

\section{Results}

Collinearity error was measured with the aid of a computer-generated protractor printed on a transparent plastic sheet. Departure from collinearity, designated as $\psi$, was measured as an angle relative to a line that was, in fact, collinear to the test segment-what we generally call its "true projection." For convenience, an angular convention was adopted in which the typical direction of error was given a sign that corresponded to the sign of theta. Specifically, for the upper right and lower left quadrants, a counterclockwise departure from true projection was scored as a positive $\psi$ error, and for the other two quadrants, a clockwise departure was defined as positive. The error on each page was measured with an accuracy of $.5^{\circ}$, and for each combination of treatment conditions, the subject's scores in the four quadrants were averaged and rounded to $.1^{\circ}$. Using a two-way, within-subjects analysis of variance, the orientation of the induction segment (Factor B, angle) was found to have a significant influence on collinearity judgments $[F(6,42)=33.3, p<$ $.0001]$. However, length of the test segment (Factor A) did not yield a significant difference $[F(3,21)=0.33, p<$ $.80]$, nor did the $\mathrm{A} \times \mathrm{B}$ interaction $[F(18,126)=1.22$, $p<.26]$.

The mean across subjects for each treatment combination has been plotted in Figure 2. Overall the angular induction effect appears to be the same for each of the four lengths of test segment, being strongest at $15^{\circ}$ and declining more or less linearly as the angle is increased. This has been the pattern of effect in other studies from this laboratory done with long test segments with various configurations of the induction element (Greene, in press; Greene \& Pavlov, 1989). The present results indicate that the induction effects are comparable even when the test

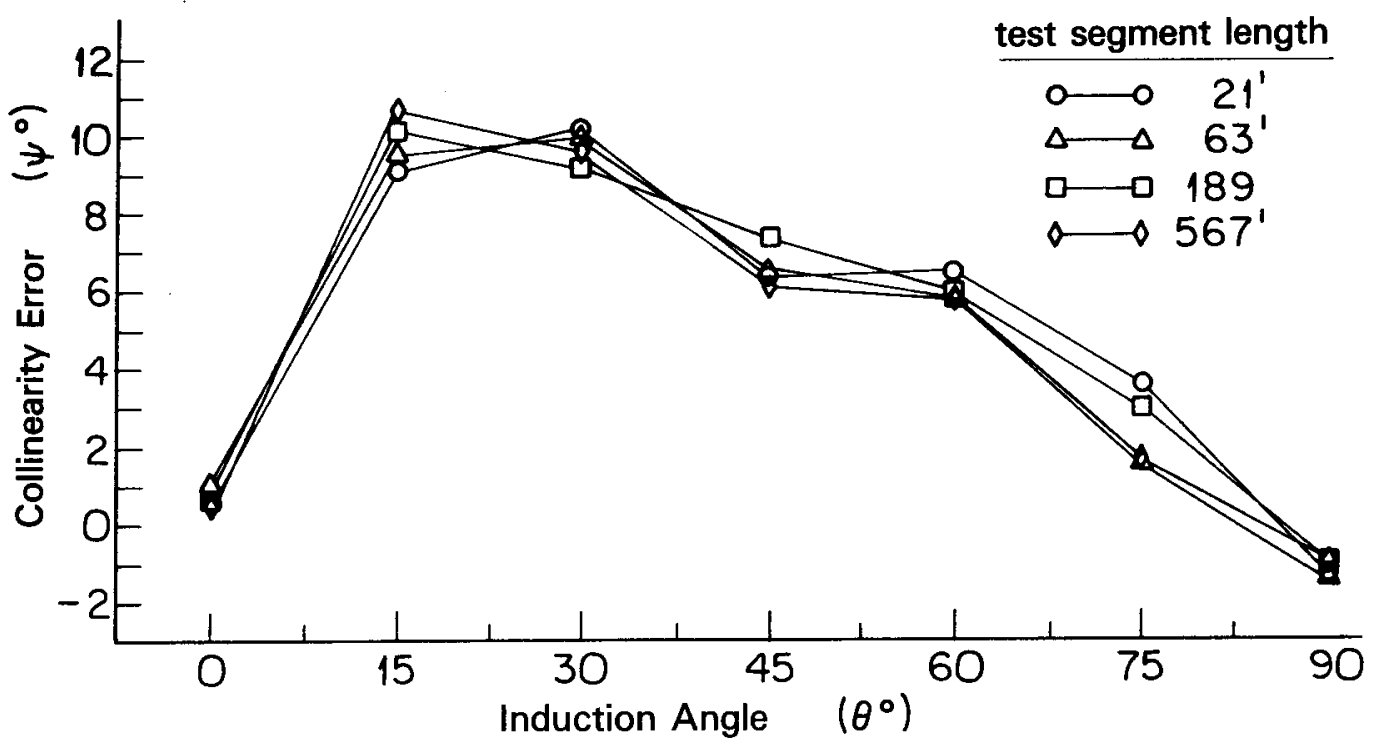

Figure 2. Collinearity errors appeared to be linear across most of the induction angles - that is, $\theta$. The induction effect was maximal at or near $15^{\circ}$, irrespective of the length of the test segment. 
segment is extremely short-specifically, $21^{\prime}$ of visual angle-which is within the range used by Bouma and Andriessen (1970). It is unlikely, therefore, that the length of the test segment explains the discrepancy between the results of this laboratory, which show the strength of effect peaking at $15^{\circ}$ or closer, and the demonstration by Bouma and Andriessen of peak effects at $45^{\circ}$.

\section{EXPERIMENT 2}

\section{Rationale}

A number of studies suggest that in addition to the basic induction process, wherein the orientation of one segment has an impact on perceived orientation and collinearity judgments of another, there is a second factor that relates to the orientation of the material relative to the observer. Judd (1899) appears to have been the first to describe the relative weakness of the Poggendorff illusion when the configuration is rotated so that collinear segments are placed at the vertical or horizontal position. Obonai (1931), Leibowitz and Toffey (1966), and Weintraub and Krantz (1971) have provided quantitative confirmation of this effect, and it has been attributed to "horizontalvertical assimilation" (Hotopf, Ollerearnshaw, \& Brown, 1974) or to "horizontal-vertical preference orientations" (Bouma \& Andriessen, 1970), or it has been subsumed under the headings of the "oblique effect" (Appelle, 1972) or "visual anisotropy" (Howard, 1982). Bouma and Andriessen's (1970) analysis is of particular interest, for they hypothesized that neurons that detect vertical and horizontal lines are more sensitive than those that register oblique lines. Because of this greater sensitivity, one would predict that the judgment of isolated line segments would be biased toward a cardinal axis (which Bouma \& Andriessen previously confirmed in 1968), and also that an induction line that is horizontal or vertical has a greater effect on judgment. Bouma and Andriessen's model was formulated to account for the finding of stronger bias where the induction segment was $45^{\circ}$ relative to the test segment, thereby bringing it into alignment with one of the cardinal axes.

Alternatively, one might hypothesize that the accuracy of mental projection is affected by the orientation of the test segment. It is possible that collinearity judgments are most accurate when the test segment is aligned with the cardinal axes and are least accurate (and thus most accessible to influence) when it lies at $45^{\circ}$. Rotating the entire stimulus configuration, as was done by Judd (1899), Obonai (1931), Leibowitz and Toffey (1966), and Weintraub and Krantz (1971), serves to confound these issues. Greene (in press) has evaluated and modeled the strength of induction under conditions that separate the two factors, and the results of that study suggest that the process should be seen as an interaction of the two-a primary induction that is determined by the relative angle between the test and induction segments, and a secondary influence that is a function of the orientation of the test segment relative to the observer.
This discourse is pertinent to the present question of where "peak effects" will be seen, because the methods of Bouma and Andriessen (1970) also contain this confound. They found their key results-that is, curves of collinearity error showing peaks at $45^{\circ}$-when they rotated the entire configuration (thereby keeping the induction angle constant), or when they aligned the induction segment with a cardinal axis and varied the orientation of the test segment. Both these methods alter the orientation of the test segment relative to the observer, providing both the primary induction effect and the secondary modulating effect. If the induction angle is kept constant, the size of the effect should be at a minimum when the test segment is aligned with a cardinal axis and should be maximal when it is moved to an oblique position. Where the induction segment is fixed at a cardinal orientation, rotation of the test segment will generate a curve that is the product of the primary and secondary effects and thus would be expected to peak at or near $45^{\circ}$.

To confirm the validity of this analysis, a second experiment was conducted to compare the methods that are standard for our laboratory with one of the methods used by Bouma and Andriessen (1970). These results were evaluated with the use of the Greene (in press) model, described below.

\section{Induction Model}

The quantitative model should be considered to be tentative, for a number of anomalies and individual differences in the data still indicate that we have not identified all elements of the phenomenon. As indicated above, the model calls for two main factors: a primary induction influence whose strength appears to be a linear function of the relative angle between the induction segment and the test segment $(\theta)$, and a secondary modulating factor that is a function of the orientation of the test segment relative to the observer $(\Omega)$. For any constant orientation of the test segment, the primary induction factor is seen in isolation, and the collinearity error can be expressed in radians as a simple function of pi, or in degrees:

$$
\psi^{\circ}=p\left(1-\frac{\theta^{\circ}}{90}\right) .
$$

This equation specifies a linear relation between the induction angle and collinear error, with a single free parameter $-p-$ controlling the strength of the effect. The equation calls for induction to be greatest at close angles, placing no limit on this proximity. Greene (in press) found good agreement with the model using angles as close as $7.5^{\circ}$, but the effect can peak at $15^{\circ}-30^{\circ}$ for some subjects. It is expected that a comprehensive model of angular induction will be able to delimit the range in which the effects are linear, but for the present purposes, we ignore this issue.

The secondary modulating factor $-q(\Omega)$-is a function of the relative orientation of the observer and the test segment. Previous results (Greene, in press) indicate that the 


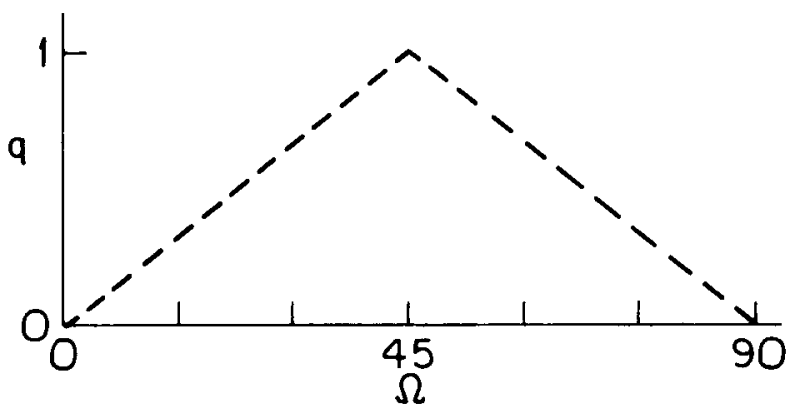

Figure 3. On the basis of previous results, our model predicts that the orientation of the test segment relative to the observer provides a function that modulates the basic induction effect. This factor, $q(\Omega)$, is thought to be piece-wise linear, being near zero strength when the test segment has a vertical or horizontal orientation, and growing to a maximum when it is $45^{\circ}$ relative to the observer.

strength of $q$ drops as a linear function of angle, being maximal with a $45^{\circ}$ test segment and decreasing to a minimum of about $15 \%$ of this strength when the test segment is moved to the vertical orientation. It is presumed that a symmetrical decline occurs in relation to the horizontal axis, providing a piece-wise linear function as shown in Figure 3. It seems premature to specify the fractional strength of parameters without further study; therefore, the present analysis assumes that the value of $q$ declines to zero. For the function shown in Figure 3, a formula can be written with the angle being expressed in radians or degrees; the latter is provided by Equation 2.

$$
q\left(\Omega^{\circ}\right)= \begin{cases}\frac{\Omega^{\circ}}{45}, & \text { if } 0 \leq \Omega^{\circ} \leq 45 \\ 2-\frac{\Omega^{\circ}}{45}, & \text { if } 45 \leq \Omega^{\circ} \leq 90\end{cases}
$$

Whenever the orientation of the test segment is varied, one uses a combination of the two factors to predict the overall induction effect. Equation 3 gives the composite formula, this being simply the product of the two factors. Note that $p$ is a constant multiplier that is independent of $\theta$, while $q$ is a function of the $\Omega$ angle.

$$
\psi^{\circ}=q\left(\Omega^{\circ}\right) \cdot p\left(1-\frac{\theta^{\circ}}{90}\right)
$$

\section{Method}

Experiment 2 was conducted to evaluate the differential effects of a fixed versus variable test segment, and to test the predictive power of the model, as discussed below. The length of the test segment was set at $448^{\prime}$, and all other stimulus dimensions were the same as for the first experiment. There were two treatment conditions, one in which the test segment remained at $45^{\circ}$ and the induction segment was varied in $7.5^{\circ}$ increments through $90^{\circ}$ following the convention described previously. For the second condition, the induction segment was fixed at the horizontal axis, and the test segment was varied in $7.5^{\circ}$ increments through $90^{\circ}$. Eight subjects, one of whom had been tested before, made collinearity judgments under conditions that were identical to the first experiment.

\section{Results}

The goal of the second experiment was to determine whether the observed data could be predicted by the model, and in particular whether the peak effects reported by Bouma and Andriessen (1970) could be derived from the collinearity judgments in which the test segment was fixed. As indicated above, where the test segment is positioned at $45^{\circ}$, the size of the induction effect is expected to be at the maximum value of $q$-that is, at unity-and thus should be a linear function of theta multiplied by the value of $p$, as specified in Equation 1. Where there is no induction segment-namely, at the $0^{\circ}$ position-the equation does not apply and the error is assumed to be zero.

To make it clear how the model was applied to the data, it may be helpful to respecify the relation of the dependent induction $(\psi)$ effect to the independent value arrays, as shown in Equations 4-6. The indices $i$ and $j$ are used to designate the individual subjects and angles, respectively. For each value of angle-ignoring $0^{\circ}$ where the model does not apply-each subject responds with a collinearity error that is dependent on the value of $p$. Although $p$ can be described as being a constant in the context of an ideal induction equation, one would expect to see variation in the effect, and it is convenient to treat $p$ as a normal random variable using the subscripts $i$ and $j$. One can solve for $p_{i j}$ as shown in Equation 5, and with this equation one uses the observed collinearity errors at each angle to derive the array of the $p$ variable. The values of $p_{i j}$ were averaged across all angles and subjects to provide an estimate of the strength of induction for the group, as shown in Equation 6.

$$
\psi_{i j}^{\circ}=p_{i j}\left(1-\frac{\theta_{j}^{\circ}}{90}\right) \quad \begin{aligned}
\text { for } i & =1 \text { to } n, \text { the number of subjects, } \\
j & =1 \text { to } m, \text { the number of angles. }
\end{aligned}
$$

$$
\begin{aligned}
& p_{i j}=\frac{\psi_{i j}^{\circ}}{\left(1-\frac{\theta_{j}^{\circ}}{90}\right)} \\
& p_{. .}=\frac{\sum_{j=1}^{m} \sum_{i=1}^{n} p_{i j}}{m n}
\end{aligned}
$$

Collinearity judgments for the fixed test segment condition-that is, $\Omega=45^{\circ}$-were processed to derive $p_{. .}$, and this value was used to predict a theoretical curve of induction effect across angles. The observed and predicted values are plotted in Figure 4.

A goodness-of-fit analysis was calculated by using a Pearson chi-square statistic, eliminating the values at $0^{\circ}$ and $90^{\circ}$ where division by zero would be required. For this analysis, one would look for a significant difference between the data and the predictions derived from the theoretical model. The $\chi^{2}=6.85$ was far from significant, with more than $50 \%$ of the distribution falling above the 


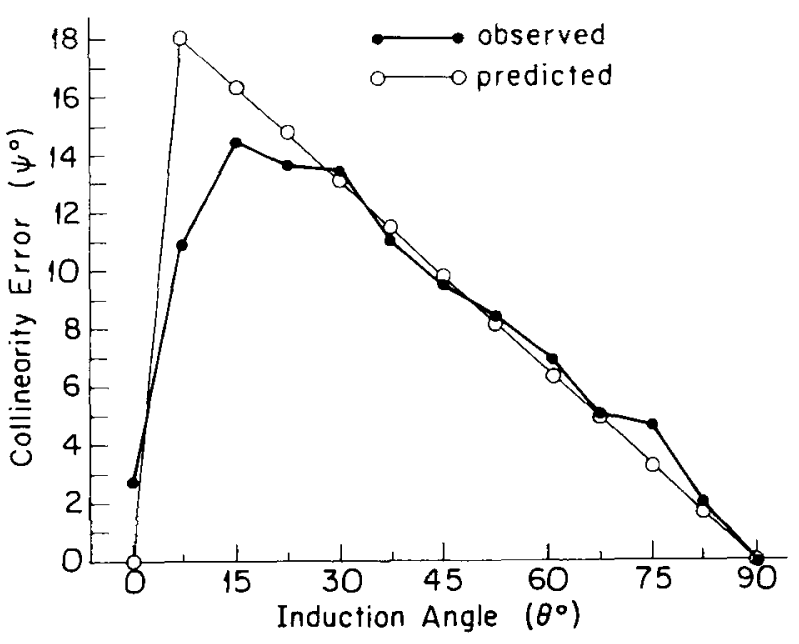

Figure 4. In half the trials of the second experiment, the relative angle of the inducing segment $(\theta)$ was varied about a test segment that was positioned at $\Omega=4^{\circ}$. Here, one sees the basic induction effect, which was a linear function of $\theta$.

calculated value. From inspection of Figure 4, it is clear that the linear effects (which the model predicts for these test conditions) hold up well through most of the range of orientations, with the induction influence deteriorating at the closer angles.

The $p_{\text {.. }}$ value - that is, the mean estimate of induction strength for the fixed test segment condition-was then used to predict responses under Bouma and Andriessen's (1970) protocol, in which the test segment was varied while the induction segment was held in a horizontal position. The appropriate model is given by Equation 3, and the observed and predicted values are plotted in Figure 5. Goodness-of-fit was evaluated, again eliminating $0^{\circ}$ and $90^{\circ}$, and the $\chi^{2}=7.68$ was not significant. As was true for the first half of the experiment, more than $50 \%$ of

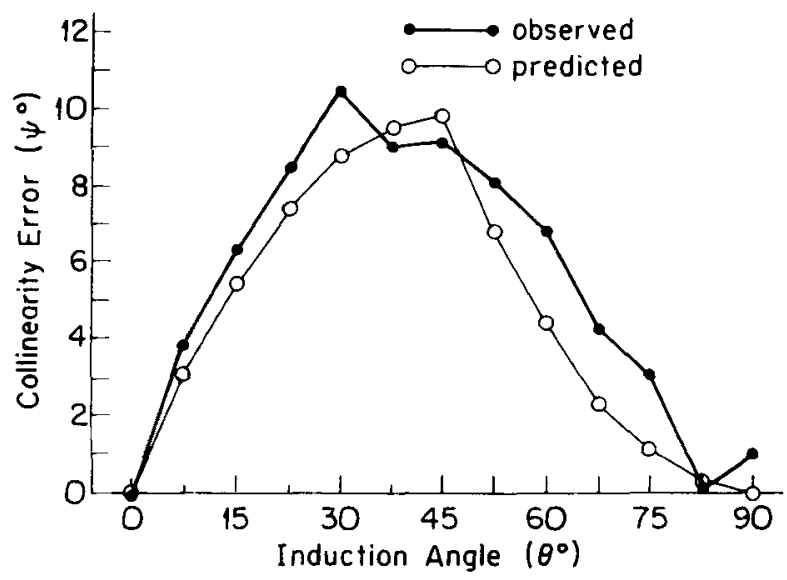

Figure 5. In the remaining trials of the second experiment, the induction segment was kept at a horizontal orientation, and the test segment was varied. Here $\theta$ equaled $\Omega$, and one can see the combined influence of the inducing and modulating factors, which resulted in a pattern of collinearity errors with a peak near $45^{\circ}$. the distribution fell above this value. These results indicate that the observed data are in substantial agreement with the predictions derived from the model.

It should be noted that the present model has been kept simple, the goal being to provide a basic framework for analysis and discussion rather than to construct an optimal fit to the data. The model allows only one free parameter ( $p$ ) for predicting strength of effect when there are two factors operating. The ultimate goal would be to gather data that would delineate boundary conditions better and specify how they should be applied.

On the basis of the present results, several problems deserve brief comment. First, the model does not predict any angular induction where the test segment is presented alone, but a source of collinearity bias may be present nonetheless. In both experiments, judgments of the isolated test segment were $1^{\circ}-3^{\circ}$ above zero, as shown in Figures 2 and 4 . This may be due to assimilation of the test segment toward a cardinal axis, as discussed below. Second, the model appears to be most adequate in the range from $90^{\circ}$ through $30^{\circ}$, with the strength at closer angles showing signs of decay (see Figure 4). Additional work is needed to quantify the parameters of this collapse. Third, it is likely that the $q(\Omega)$ portion of the model (Equation 2) will be improved once there is better quantification of the residual modulation where the test segment is aligned with a cardinal axis. Greene (in press) estimated this residual to be about $15 \%$, which is to say that the modulation strength was maximal at $45^{\circ}$ and then decreased as a linear function of omega to $15 \%$ of that value. If one uses this correction, the predictions from the model are a much better fit to the data shown in Figure 5. Finally, it is not clear why the error curve shown in Figure 5 peaks at $30^{\circ}$ rather than at $45^{\circ}$ as predicted by the model. This may be due to horizontal/vertical assimilation of the entire configuration-biasing the perceived orientation of the induction segment as well as the test segment. Alternatively, the modulation may peak at an orientation that varies for different individuals, with the location of this peak being due to chance sampling. It is also possible that the mean value at $30^{\circ}$ does not properly reflect the true induction effect at that angle. It lies about a degree (of error) above the prediction, and about three fourths of a degree above the values at $37.5^{\circ}$ and $45^{\circ}$. This small deviation from the overall curve may be an anomaly.

It is remarkable, nonetheless, how well the simple twofactor model accounts for the Figure 5 data, especially given the fact that there were no free parameters. A casual survey of the literature suggests that most theorists allow themselves at least three to four free parameters when modeling data; by adjusting their value, one can greatly improve the fit between prediction and data. However, the goal from the outset of this experiment was to derive an estimate of $p$ from the task that kept the test segment in a fixed position, and then use this value in Equation 3 to predict performance when the orientation of that segment was varied. This equation specifies the strength of effect as a function of the relative angle between the test 
segment and the induction segment, and as a function of the orientation of the test segment relative to the observer. It has only one free parameter $(p)$, its value being specified by the earlier data. With two separate factors, and without adjustment of parameters to improve the fit, the degree of match shown in Figure 5 is gratifying.

\section{DISCUSSION}

The present experiments were designed to clarify why some laboratories have found that the strength of angular induction is greatest when the biasing segment is vertical or horizontal relative to the observer, whereas other laboratories have not observed stronger effects when the induction segment is aligned with a cardinal axis. Wenderoth et al. (1979) suggested that such differences were due to the length of the segment being judged, as well as to the method for assessing its orientation. Using long vertical test segments and relative angles of $15^{\circ}, 45^{\circ}$, and $75^{\circ}$, they found a somewhat linear pattern of declining strength whether the subject was required to judge collinearity between the test segment and a dot, to set another line parallel to the test segment, or to move the test segment until it appeared to be vertical. With short test segments, they observed a similar pattern of effects for the latter two test methods, but with collinearity judgment, the strongest effect was seen at $45^{\circ}$. On the basis of the discrepancy of this point, along with the report by Bouma and Andriessen (1970) of peak induction effects at $45^{\circ}$ under similar test conditions, they hypothesized that angular induction was manifested differently for short test segments and with collinearity as the basis for judgment.

In the first experiment, four lengths of test segment were tested, the shortest being in the range of stimuli used by Bouma and Andriessen (1970), and with a sufficient number of induction angles to properly evaluate the pattern of effect. We found the greatest induction at close angles, with a fairly linear decline of strength as the relative angle between the induction segment and the test segment was increased to $90^{\circ}$. It seems likely, therefore, that the $45^{\circ}$ mean reported by Wenderoth et al. (1979) is in error. Except for that one data point, their results indicate a declining strength of induction as a function of theta, whether the test segment is short or long and whether the bias is measured using collinearity, setting a comparison line to be parallel, or setting a test segment to the vertical position.

Wenderoth et al.'s (1979) results, however, do show a differential strength of effect that depends on the method of judgment, in that collinearity errors are consistently larger than those observed using the other two methods. Similarly, Carpenter and Blakemore (1973) reported that with the tilt illusion, where the induction bias is measured by setting a comparison line to be parallel to the test segment, the maximal effect was only $2^{\circ}-3^{\circ}$. Except for this differential magnitude of influence (and a trivial difference in how the data was plotted), Carpenter and Blakemore's results are very similar to the effects that we have observed when using collinearity judgment (see Greene, in press, and Greene \& Pavlov, 1989, as well as present findings). In addition, using a variation of the Poggendorff in which the induction lines formed a right angle, Greene (1988) reported that the illusion was manifested as a differential tilt of the two oblique segments. The size of the judgment error was quantitatively evaluated and found to be about $3^{\circ}$.

It would appear that the induction process causes the orientation of the test segment to be misjudged, but it is not clear why collinearity errors are many orders of magnitude larger than the misperceived orientation of the test segment. If one assumes that the point selected as being collinear is misplaced because of the bias in orientation of the test segment, then the amount of error should be the same. It is conceivable that setting a line to be parallel underestimates the full effect because of a concomitant bias of the induction line upon the comparison line. If this argument is correct, the true bias for the tilt illusion should be about $10^{\circ}-15^{\circ}$ (depending on theta and other stimulus factors), but this bias is not reflected by the adjustment of a comparison line, because the perceived orientation of that line is also affected by the induction process. This would not explain, however, why one gets a $2^{\circ}-3^{\circ}$ maximal bias when the subject is asked to adjust a segment so that it appears to be vertical.

As an alternative, it is possible that the extrapolation of an imaginary line across open space is a separate process that is susceptible to angular induction. According to this view, the induction segment would affect the orientation of the test segment, and also (to a greater degree) the process of extending an imaginary line across space to potential target sites. Some support for this view comes from the report of Wenderoth, Beh, and White (1978), who found that collinearity bias for a point placed in the unobstructed path of the test segment (from the end that was not in contact with the induction line) was several times smaller than the level of bias from the end that was in contact with the induction segment. As these authors argued, the projection error cannot be attributed completely to misperceived tilt of the test segment, for if this were the case, the effects should be the same irrespective of the end at which the dot was placed.

From the present data we cannot resolve all the questions about the various manifestations of angular induction. It may be more productive to focus on the initial question of why some studies, especially that of Bouma and Andriessen (1970), have reported peak induction effects where the relative angle between the induction and test segments was at $45^{\circ}$, whereas other work has shown the strength peaking at much closer angles. The results of the second experiment suggest that this problem is due to procedural differences. When the test segment is kept at a fixed position, the basic induction effect will be manifested, but when its orientation is changed with respect to the observer, a second modulating factor comes into play. Under the influence of this modulation, angular induction produces larger errors if the test segment lies at an oblique orientation, and much smaller errors as the test segment approaches one of the cardinal axes. 
It should be said, however, that the behavioral data are intelligible only within the context of one's interpretation. It is pertinent, therefore, that my view of the induction process differs from the one advanced by Bouma and Andriessen (1970). Their model calls for two sources of influence on the perceived orientation of the test segment: (1) a bias toward an axis that lies perpendicular to the induction segment, and (2) a bias toward the horizontal or vertical axis, depending on which is closest to the orientation of the test segment. They implement and describe their model within the context of neural orientation sensors, the neurophysiology for this approach being provided by Hubel and Wiesel $(1962,1965,1968)$, with psychological application being suggested by MacKay (1965), Day (1965), Ganz (1966), Andrews (1967a, 1967b), Robinson (1968), and their own earlier work with misperception of isolated line segments (Bouma \& Andriessen, 1968).

The first element of their model corresponds to what I have described as the "basic induction effect." There does not seem to be any real difference between Bouma and Andriessen (1970) and myself as to the predicted direction of this bias, though there are some disparities in how the influence is described and the degree to which the effects should be attributed to misperceived orientation of the test segment. The least important difference occurs between my description of the bias as being produced by the orientation of the induction segment relative to the test segment, and their suggestion that the bias is toward the perpendicular axis of the induction segment. It is conceivable that the distinction between repulsion by a segment and attraction by the orthogonal of that segment might become meaningful within the context of a specific neural model, but, for the present, I am inclined to see this as a semantic distinction. With respect to the basic induction process, however, it should be noted that they attribute all of the collinearity error to misperception of the tilt of the test segment. As discussed above, it is likely that some of the error is due to an influence on the projection process.

The second element of their model, a bias of perceived orientation of the test segment that is produced by the horizontal or vertical axis of the observer, derives from their earlier work (Bouma \& Andriessen, 1968). In that study, they found collinearity errors when subjects were asked to judge isolated test segments, and they interpreted the effect as an erroneous judgment of the segment as lying closer to the horizontal or vertical. This is one of a number of manifestations of the "oblique effect," a term used by Appelle (1972) to describe differentials of perception and physiology as a function of stimulus orientation.

Viewed as an isolated influence, such horizontalvertical assimilation should not greatly complicate the issue. Bouma and Andriessen (1968) generally observed the size of the error to be about $3^{\circ}$, which is consistent with other reports (see Howard, 1982). Similarly, previous research in this laboratory has often found a $1^{\circ}-3^{\circ}$ collinearity error for the isolated test segment, and when the induction segment was at the $90^{\circ}$ (null-influence) po- sition. In fact, in one experiment of the Greene (in press) study, the entire induction curve appeared to be shifted up several degrees when the test segment was at $30^{\circ}$ to the vertical, and down by the same amount when it was positioned at $60^{\circ}$. It is conceivable, therefore, that assimilation adds a small constant bias to judgments of a test segment irrespective of the orientation of the induction segment.

The difficulty, however, comes with the specific neural model which is advanced by Bouma and Andriessen (1970). This model calls for a differential sensitivity of the orientation detectors as the basis for the cardinal-axis bias, which then requires that a cardinal axis will influence the strength of the primary induction process. In other words, having specified that horizontal and vertical detectors have greater sensitivity, one must find a differential influence of the induction segment when it aligns with one of these axes. The present studies argue against this possibility. As can be seen in Figure 2 from Experiment 1, and Figure 4 from Experiment 2, there is no indication of increased strength of effect at the point where the induction line lies vertical relative to the observerthat is, at the $45^{\circ}$ angle.

Nonetheless, with some stimulus configurations, the manipulation of relative angle can produce differential effects that peak at about $45^{\circ}$. What is the source of this pattern? It is likely another facet of the "oblique effect." Many studies support the view that judgments are better when the stimulus to be evaluated is aligned with the horizontal or vertical axis of the observer (Higgens \& Stultz, 1948, 1950; Liebowitz, 1953; Ogilvie \& Taylor, 1958; Taylor, 1963). Attneave and Olson (1967) reported that identification of nonfoveal, briefly flashed lines was more rapid and accurate when they were horizontal or vertical. Andrews (1967b) found evidence of better accuracy whether long or short exposure times were used. Other manifestations of the oblique effect have been found with various stimulus and task conditions (Berkley, Kitterle, \& Watkins, 1975; Campbell, Kulikowski, \& Levinson, 1966; Orban, Vandenbussche, \& Vogels, 1984; Vogels, Orban, \& Vandenbussche, 1984). Appelle (1972) and Howard (1982) have provided insightful reviews of the various manifestations of these effects in humans and animals.

The relevance of these reports occurs in the suggestion that orientation judgment is more accurate and less variable when the segment to be evaluated is aligned with a cardinal axis, and more so when it is tilted toward the oblique. It may be reasonable to suggest that the greater stability of responses provides an offset to the induction bias. In other words, the angular influence may be manifested relative to the variability that is found at a given orientation of the test segment-perhaps as a constant multiple of the standard deviation of the judgment process. When that variability is low-namely, when the test segment is aligned with a cardinal axis-the induction effect would be commensurately lower. When the variability of the judgment process is high, as with a test segment that lies at an oblique orientation, the bias would appear as 
a larger error. The suggestion, therefore, is that the modulating factor that is described quantitatively by Equation 2 reflects the relative susceptability of the judgment system to the angular induction effect.

With respect to neural orientation detectors, this hypothesis corresponds to the notion that the width of the tuning curve is smaller for the cells that respond to the horizontal and vertical. The evidence for this possibility has been reviewed by Howard (1982) and related issues have been discussed by Orban et al. (1984), Mustillo, Francis, Oross, Fox, and Orban (1988), and Heeley and Buchanan-Smith (1990).

\section{REFERENCES}

ANDREws, D. P. (1967a). Perception of contour orientation in the central fovea: Part I. Short lines. Vision Research, 7, 975-997.

ANDREws, D. P. (1967b). Perception of contour orientation in the central fovea: Part II. Spatial integration. Vision Research, 7, 999-1013.

APPELLE, S. (1972). Perception and discrimination as a function of stimulus orientation: The "oblique effect" in man and animals. Psychological Bulletin, 78, 266-278.

ATTNEAVE, F., \& Olson, R. K. (1967). Discriminability of stimuli varying in physical and retinal orientation. Journal of Experimental Psychology, 74, 149-157.

Bacon, J., \& KING-Smith, P. E. (1977). The detection of line segments. Perception, 6, 125-131.

Berkley, M. A., Kitterle, F., \& Watkins, D. W. (1975). Grating visibility as a function of orientation and retinal eccentricity. Vision Research, 15, 239-244.

Bouma, H., \& ANDRIESSEN, J. J. (1968). Perceived orientation of isolated line segments. Vision Research, 8, 493-507.

Bouma, H., \& ANDRIESSEN, J. J. (1970). Induced changes in the perceived orientation of line segments. Vision Research, 10, 333-349.

Camprell, F. W., Kulikowski, J. J., \& Levinson, J. (1966). The effect of orientation on the visual resolution of gratings. Journal of Physiology, 187, 427-436.

Carpenter, R. H. S., \& Blakemore, C. (1973). Interactions between orientations in human vision. Experimental Brain Research, 16, 287-303.

DAY, R. H. (1965). Inappropriate constancy explanation of spatial distortions. Nature, 207, 891-893.

GANZ, L. (1966). Mechanism of the figural aftereffects. Psychological Review, 73, 128-150.

Greene, E. (1988). The comer Poggendorff. Perception, 17, 65-70.

GReENE, E. (1993). The influence of response factors on the magnitude of angular induction. Perceptual \& Motor Skills, 76, 323-335.

GreENE, E. (in press). Collinearity judgment as a function of induction angle. Perceptual \& Motor Skills.

GreENe, E., \& PAVLov, G. (1989). Angular induction as a function of contact and target orientation. Perception, 18, 143-154.

Heeley, D. W., \& Buchanan-Smith, H. M. (1990). Recognition of stimulus orientation. Vision Research, 30, 1429-1437.

Higgins, G. C., \& STULtz, K. (1948). Visual acuity as measured with various orientations of a parallel-line test object. Journal of the Optical Society of America, 38, 756-758.

HigGins, G. C., \& STULTZ, K. (1950). Variation of visual acuity with various test-object orientations and viewing conditions. Journal of the Optical Society of America, 40, 135-137.

Hotopf, W. H. N., Ollerearnshaw, C., \& Brown, S. (1974). The regression to right angles tendency and the Poggendorff illusion: III. British Journal of Psychology, 65, 213-231.

How ARD, I. P. (1982). Human visual orientation. New York: Wiley.

Hubel, D. H., \& Wiesel, T. N. (1962). Receptive fields, binocular interaction, and functional architecture in the cat's visual cortex. Journal of Physiology, 160, 106-154.

Hubel, D. H., \& Wiesel, T. N. (1965). Receptive fields and functional architecture in two nonstriate visual areas (18 and 19) of the cat. Journal of Neurophysiology, 28, 229-289.

Hubel, D. H., \& Wiesel, T. N. (1968). Receptive fields and functional architecture of the monkey striate cortex. Journal of Physiology, 195, 215-243.

JudD, C. H. (1899). A study of geometrical illusions. Psychological Review, 6, 241-261.

LEIBowITZ, H. (1953). Some observations and theory on the variation of visual acuity with the orientation of the test object. Journal of the Optical Society of America, 43, 902-905.

LEIBOWITZ, H., \& ToFFEY, S. (1966). The effect of rotation and tilt on the magnitude of the Poggendorff illusion. Vision Research, 6, 101-103.

MacKay, D. M. (1965). Visual noise as a tool of research. Journal of General Psychology, 72, 181-197.

Mustillo, P., Francis, E., Oross, S., Fox, R., \& Orban, G. A (1988). Anisotropies in global stereoscopic orientation discrimination. Vision Research, 28, 1315-1321.

OBONAI, T. (1931). Experimentelle Untersuchungen über den Aufbau des Sehraumes. Archiv für die gesamte Psychologie, 82, 308-328.

OgILVIE, J. C., \& TAYLOR, M. M. (1958). Effect of orientation on the visibility of fine wires. Journal of the Optical Society of America, 48, 628-629.

Orban, G. A., Vandenbussche, E., \& Vogels, R. (1984). Human orientation discrimination tested with long stimuli. Vision Research, 24, 121-128.

RoBINSON, J. O. (1968). Retinal inhibition in visual distortion. British Journal of Psychology, 59, 29-36.

TAYLOR, M. M. (1963). Visual discrimination and orientation. Journal of the Optical Society of America, 53, 763-765.

Tyler, C. W., \& NaKayama, K. (1984). Size interactions in the perception of orientation. In L. Spillmann \& B. R. Wooten (Eds.), Sensory experience, adaptation, and perception (pp. 529-546). Hillsdale, NJ: Erlbaum.

Vassilev, A., \& Penchev, A. (1976). Spatial and temporal summa tion in the perception of lines. Vision Research, 16, 1329-1335.

Vogels, R., Orban, G. A., \& VANDENBussche, E. (1984). Meridonal variations in orientation discrimination in normal and amblyopic vision. Investigative Ophthalmology \& Visual Science, 25, 720-728.

Weintraub, D. J., \& Brown, S. (1986). Perceptual plane geometry: Collinearity judgments probe the perceived orientation of an angle's sides. Journal of Experimental Psychology: Human Perception \& Performance, 12, 434-444.

Weintraub, D. J., \& Krantz, D. H. (1971). The Poggendorff illusion: Amputations, rotations, and other perturbations. Perception \& Psychophysics, 10, 257-264.

Wenderoth, P., Beh, H., \& White, D. (1978). Perceptual distortion of an oblique line in the presence of an abutting vertical line. Vision Research, 18, 923-930.

Wenderoth, P., Parkinson, A., \& White, D. (1979). A comparison of visual tilt illusions measured by the techniques of vertical setting, parallel matching, and dot alignment. Perception, 8, 47-57.

(Manuscript received February 24, 1992; revision accepted for publication April 22, 1993.) 